\title{
CONSTRAINING THE PHYSICS OF AM CANUM VENATICORUM SYSTEMS WITH THE ACCRETION DISK INSTABILITY MODEL
}

\author{
John K. CAnnizzo ${ }^{1,2}$, Gijs Nelemans ${ }^{3,4}$ \\ ApJ, in press - Received 2014 December 15; accepted 2015 February 4
}

\begin{abstract}
Recent work by Levitan et al has expanded the long-term photometric database for AM CVn stars. In particular, their outburst properties are well-correlated with orbital period, and allow constraints to be placed on the secular mass transfer rate between secondary and primary if one adopts the disk instability model for the outbursts. We use the observed range of outbursting behavior for AM CVn systems as a function of orbital period to place a constraint on mass transfer rate versus orbital period. We infer a rate $\sim 5 \times 10^{-9} M_{\odot} \mathrm{yr}^{-1}\left(P_{\mathrm{orb}} / 1000 \mathrm{~s}\right)^{-5.2}$. We show the functional form so obtained is consistent with the recurrence time-orbital period relation found by Levitan et al using a simple theory for the recurrence time. Also, we predict their steep dependence of outburst duration on orbital period will flatten considerably once the longer orbital period systems have more complete observations.
\end{abstract}

Subject headings: accretion, accretion disks - binaries: close - novae, cataclysmic variables - stars: individual (AM Canum Venaticorum)

\section{INTRODUCTION}

Cataclysmic variables (CVs) are semi-detached binaries consisting of interacting stars in which a Roche-lobe filling secondary transfers matter to a more massive, and also more compact primary. The dwarf novae (DNe) constitute a subclass further characterized by semiregular outbursts of several magnitudes, recurring on timescale of days to years (Warner 1995a). The novalikes reside at higher mass transfer rates and do not show DN outbursts. The limit cycle accretion disk instability model (DIM) has been successful in explaining the outbursts (Smak 1984). CVs evolve to shorter orbital periods, driven by angular momentum loss (AML) from a combination of a magnetic wind from the secondary star and gravitational radiation (Knigge, Baraffe, \& Patterson $2011=\mathrm{KBP}$ ). Although the DIM is generally agreed to be the correct explanation for DN outbursts, mainly because the observed dividing line between steady and outbursting systems (i.e., DNe and novalikes) agrees with theory (Smak 1983b), further progress has come slowly. For instance, any theory for the recurrence time for DN outbursts (Cannizzo, Shafter, Wheeler $1988=$ CSW) is muddied by the fact that the outburst properties at a given orbital period exhibit a wide scatter (Warner 1995a).

The AM CVn stars are a subset of CVs at very short orbital period, less than an hour, whose spectra are dominated by helium (Nelemans 2005, Solheim 2010). The prototype, AM CVn, was discovered neary fifty years ago (Smak 1967). Paczyński (1967) proposed the system to be a short orbital period binary with two degenerate, He-rich stars with a period evolution driven by gravitational wave radiation (GWR), but he considered detached rather than semi-detached binaries. Motivated by observational inferences of mass transfer in AM CVn, namely rapid photometric flickering (Warner \&

\footnotetext{
${ }^{1}$ CRESST and Astroparticle Physics Laboratory, NASA/GSFC, Greenbelt, MD 20771, USA; John.K.Cannizzo@nasa.gov

${ }^{2}$ Department of Physics, University of Maryland, Baltimore County, 1000 Hilltop Circle, Baltimore, MD 21250, USA

${ }^{3}$ Department of Astrophysics/IMAPP, Radboud University Nijmegen, P.O. Box 9010, 6500 GL Nijmegen, The Netherlands

${ }^{4}$ Institute for Astronomy, KU Leuven, Celestijnenlaan 200D, 3001 Leuven, Belgium
}

Robinson 1972), Faulkner et al. (1972) presented the first self-consistent model for AM CVns, correctly taking into account their semi-detached nature.

The coincidence in stable periodic variations in spectral lines with photometric variations can sometimes be used to obtain orbital periods, and the weak X-ray emission indicates a white dwarf (WD) primary accretor rather than a neutron star (Nather et al. 1981, Patterson et al. 1993, Groot et al. 2001). AM CVn stars are thought to have evolved beyond the minimum orbital period which divides nondegenerate and degenerate secondaries. The systems span a wide range in mass transfer rate $\dot{M}_{T}$. In fact, in the AM CVn stars one sees a range spanning not only the high to intermediate range equivalent to the novalike $\rightarrow$ DNe transition in normal hydrogencontaining $\mathrm{CV}$ s, but also a range spanning intermediate to low $\dot{M}_{T}$ values. For the low $\dot{M}_{T}$ systems the disk is too cool to have DIM outbursts.

In some sense the AM CVn stars are a better laboratory for the DIM than normal DNe because less scatter is expected in their $\dot{M}_{T}$ values at a given orbital period, given that GWR is the sole AML mechanism. KBP find that the normal (i.e., solar composition) short orbital period DNe below the period gap must have AML enhanced by a factor $\sim 2.47$ above that given solely by GWR in order to produce the observed period gap. This only applies to an ensemble average; as with the DNe above the gap, the outburst properties of systems below the gap show considerable scatter at a given orbital period. In addition, $\dot{M}_{T}$ varies by more than four orders of magnitude across the range of orbital periods in the AM CVn systems, which makes orbital period-dependent properties of the outbursts more noticeable.

Amassing a large database for AM CVn stars has been hampered by their faintness. Now, thanks to the results of dedicated transient surveys, an avalanche of new systems and data on outbursting behavior has become available. Levitan et al. (2015) present the results of a comprehensive study of AM CVn systems over nearly 10 yr. They present outburst data on 32 systems with known orbital periods, ranging from 5 to 65 min. A similar study with a slightly smaller sample was presented by Ramsay et al. (2012). 
In Section 2 we discuss the DIM in the context of AM CVn accretion disks. In Section 3 we look at the recurrence time for superoutbursts, and in section 4 the superoutburst duration. Section 5 summarizes our findings.

\section{THE DIM IN AM CVN SYSTEMS}

\subsection{Background}

A calculation of the vertical structure of geometrically thin disks reveals the steady state physics underlying the limit cycle behavior. the vertical structure as effective temperature $T_{\text {eff }}$ versus surface density $\Sigma$ (Meyer \& Meyer-Hofmeister 1981, Cannizzo \& Wheeler 1984). One finds a hysteretic relation between midplane temperature $T_{\text {mid }}$ and surface density $\Sigma$, or equivalently, between effective temperature $T_{\text {eff }}$ and $\Sigma$. Each radius $r$ and viscosity parameter $\alpha$ value has its own $\mathrm{S}-$ curve. A parameterization of the results of these calculations allows a determination of the surface densities at the maxima and minima in the S-curve, $\Sigma_{\max }(r, \alpha)$ and $\Sigma_{\min }(r, \alpha)$, as well as other physical quantities associated with these extrema, like midplane and effective temperature. One relates the local accretion rate to $T_{\text {eff, } m a x}$ and $T_{\text {eff,min }}$ using the standard Shakura \& Sunyaev (1973) relation

$$
\sigma T_{\mathrm{eff}}{ }^{4}=\frac{3}{8 \pi} \frac{G \dot{M} M_{1}}{r^{3}} .
$$

In the DIM, gas accumulates in quiescence and accretes onto the central object in outburst. (e.g., Cannizzo 1993a, Lasota 2001 for reviews). The phases of quiescence and outburst are mediated by the action of heating and cooling fronts that transverse the disk and bring about phase transitions between low and high states, consisting of neutral and ionized gas, respectively. During quiescence, when the surface density $\Sigma(r)$ at some radius within the disk exceeds a critical value $\Sigma_{\max }(r)$, a transition to the high state is initiated; during outburst, when $\Sigma(r)$ drops below a different critical value $\Sigma_{\min }(r)$, a transition to the low state is initiated. Low $\rightarrow$ high transitions can begin at any radius, whereas high $\rightarrow$ low transitions begin at the outer disk edge.

This situation comes about because of the following: In quiescence the disk is very non-steady so that mass accumulates in the outer regions. The surface density distribution is bounded by $\Sigma_{\max }(r)$, which increases with radius. (Both $\Sigma_{\max }(r)$ and $\Sigma_{\min }(r)$ scale close to linearly with $r$.) In the outburst disk, however, $\Sigma(r) \underset{\sim}{\sim} r^{-3 / 4}$. The accretion disk mass is conserved, i.e., the disk mass accumulated by the end of quiescence $\Delta M_{\text {cold }}$ is the same as that in the hot disk $\Delta M_{\text {hot }}$ immediately after the heating transition has occurred. Therefore there must be a substantial redistribution of $\Sigma(r)$ - from a profile $\underset{\sim}{\sim} r$ in quiescence to $\propto r^{-3 / 4}$ in outburst. Since $\Sigma(r)$ is smallest at large radii in the outbursting disk, and since $\Sigma(r)<\Sigma_{\min }(r)$ is the condition for the cooling transition to begin, cooling fronts are always initiated in the outer disk.

One can define a "maximum mass" $\Delta M_{\max } \equiv$ $\int 2 \pi r d r \Sigma_{\max }(r)$ that the disk could possibly achieve during the accumulation phase, i.e., quiescence. Obviously one cannot have $\Sigma(r)>\Sigma_{\max }(r)$ in quiescence or else the instability would have already been triggered. In practice, time dependent calculations show that the true disk mass at the end of quiescence $\Delta M_{\text {cold }}$ is typically $\sim 1 / 10-1 / 3$ of the "maximum mass" (e.g., Cannizzo 1993b). Therefore one may write $\Delta M_{\text {cold }}=f \Delta M_{\max }$, with $f \simeq 1 / 10-1 / 3$.
Levitan et al. (2015) restrict their attention to superoutbursts in their outburst statistics. These are analogous to long outbursts in DNe above the period gap (van Paradijs 1983); most of the mass accumulated during quiescence accreted onto the primary before the disk shuts off. For normal, "short" outbursts, only a few percent of the stored gas accretes onto the central object: the thermal time scale of thin disk is short compared to the viscous time scale, and the cooling front that is launched from the outer edge of the disk almost as soon as the disk enters into outburst traverses the disk and reverts it back to quiescence. For disks that have been "filled" to a higher level with respect to $\Delta M_{\max }$, the surface density in the outer disk can significantly exceed the critical surface density $\Sigma_{\min }$. In order for the cooling front to begin, however, the outer surface density $\Sigma\left(r_{\text {outer }}\right)$ must drop below $\Sigma_{\min }\left(r_{\text {outer }}\right)$. Disks in this state generate much longer outbursts, with slower "viscous" plateaus, because the entire disk must remain in its high, completely ionized state until enough mass has been lost onto the primary for the condition $\Sigma\left(r_{\text {outer }}\right)<\Sigma_{\min }\left(r_{\text {outer }}\right)$ to be satisfied.

Various studies have investigated the DIM in AM CVn systems (Smak 1983a, Cannizzo 1984, Tsugawa \& Osaki $1997=$ TO97, El-Khoury, \& Wickramasinghe 2000, Menou, Perna, \& Hernquist 2002, Lasota, Dubus, \& Kruk $2008=$ LDK, Kotko et al. $2012=\mathrm{KLDH})$. These investigations generally consist of first calculating the steady state accretion disk structure by integrating the vertical structure equations to parameterize the $\mathrm{S}$-curve relation between surface density $\Sigma(r, \alpha)$ and effective temperature $T_{\text {eff }}(r, \alpha)$, and then using scalings for the steady state physics as input into a time dependent model to calculate light curves. TO97 did not solve the full set of equations for the vertical structure but rather prescribed a functional form for the flux $F(z)$. We restrict our consideration of the scalings to the two most recent studies LDK and $\mathrm{KLDH}$, which integrate the complete set of structure equations and present a complete set of scalings for both the local minima and maxima in $\Sigma$.

As a model for the mass-losing secondaries in AM CVns, Deloye et al. (2005) calculate pseudo-evolutionary sequences for donors with varying degrees of degeneracy. In their Figure 2 they calculate tracks in the $\dot{M}_{T}-P_{\text {orb }}$ plane for four isotherms based on central donor temperatures $T_{c}$ ranging from $10^{4} \mathrm{~K}$ to $10^{7} \mathrm{~K}$, assuming a constant primary mass $0.6 M_{\odot}$. They also indicate the upper and lower bounds of the instability strip for the DIM taken from TO97. For their two lowest tracks, $T_{c}=10^{4} \mathrm{~K}$ and $10^{6} \mathrm{~K}$, the instability strip spans roughly the correct (i.e., observed) period range. Their tracks do not represent true evolutionary sequences since $T_{c}$ is taken to be constant along a track.

\subsection{Instability Criteria as Power Law Scalings}

The range of mass transfer from the secondary star feeding into the outer disk $\dot{M}_{T}$ which allows for unstable behavior, i.e., dwarf nova outbursts, is set by the local stability criteria at the inner and outer edge of the accretion disk.

KLDH calculate many $\mathrm{S}$-curves for accretion disks relevant for ultracompact binaries with no hydrogen, $X=0$. The basic finding is that the steady state scalings for the DIM are shifted to higher surface densities and temperatures. They present three sets of scalings for (i) $Y=1$, (ii) $Y=0.98$, $Z=0.02$, and (iii) $Y=0.96, Z=0.04$. Their results are given in terms of $\alpha, r$, and $M_{1}$. The range for unstable disk behavior is determined by the $\mathrm{S}$-curve for the in- 
ner and outer disk radii. If $\dot{M}_{T}>\dot{M}_{T, 2} \equiv \dot{M}_{\text {min, outer, }}$, the rate of accretion associated with the minimum in $\Sigma$ at $r_{\text {outer }}$, the disk will be stable in the high, ionized state. If $\dot{M}_{T}<\dot{M}_{T, 1} \equiv \dot{M}_{\max , \text { inner, }}$, the rate of accretion associated with the maximum in $\Sigma$ at $r_{\text {inner }}$, the disk will be stable in the low state.

The KLDH scalings are not convenient; for comparison with observations we must replace disk radius $r$ with orbital period $P_{\text {orb }}$ in the KLDH scaling for $\dot{M}_{T, 2}$, and with the primary radius in the $\dot{M}_{T, 1}$ scaling. We follow TO97 in making these conversions:

(1) For the outer scaling, $\dot{M}_{T, 2}$, we relate the outer disk radius $r_{\text {outer }}$ to orbital period using Figure 5 from van Haaften et al. (2012). We identify the Roche lobe radius $R_{L 1}$ with $r_{\text {outer }}$ and fit a simple power-law to $R_{L 1} / a$ as a function of the mass ratio $q=m_{2} / m_{1}$ The semi-major axis is $a$. Over the relevant range $0.01 \lesssim q \lesssim 0.03$ we fit $R_{L 1} / a \simeq$ $0.85(q / 0.01)^{-0.06}$. Based on the results of van Haaften et al. (2012) we adopt a fiducial secondary star $m_{2}\left(P_{\text {orb }}\right)$ relation

$$
m_{2}=0.038\left(\frac{P_{\text {orb }}}{1000 \mathrm{~s}}\right)^{-1.3}
$$

(see their Sect. 2.3), corresponding to $R_{2} \propto M_{2}^{-0.18}$, which gives, using Kepler's law,

$$
r_{o, 10}=1.18 m_{1}^{0.39}(1+q)^{1 / 3}\left(\frac{P_{\text {orb }}}{1000 \mathrm{~s}}\right)^{0.74},
$$

where $r_{o, 10}=r_{\text {outer }} / 10^{10} \mathrm{~cm}$.

(2) For the inner scaling, $\dot{M}_{T, 1}$, we adopt the standard inner boundary zero torque condition (Shakura \& Sunyaev 1973) for which the maximum effective temperature

$$
T_{\text {eff }}(\max )=0.488\left(\frac{3 G M_{\mathrm{wd}} \dot{M}}{8 \pi \sigma R_{\mathrm{wd}}}\right)^{1 / 4}
$$

is reached at 49/36 times the inner edge, taken to be the primary radius. (The "max" in this equation refers to the local radial maximum in $T_{\text {eff }}$ in a steady state disk due to the inner boundary condition, not the local maximum in $\Sigma$ in the S-curve.) Thus $\dot{M}_{T, 1}$ is enhanced by a factor $(0.488)^{-4}$ and evaluated at $(49 / 36) R_{\mathrm{wd}}$.

For the primary mass-radius relation, we fit a power law to Eggleton's scaling of a zero-temperature WD, i.e., for which the ratio of atomic number to atomic weight $Z / A=$ 1/2 (Rappaport et al. 1987, see their eqn. [19], with $\left.M_{\mathrm{Ch}}=1.44 M_{\odot}\right)$. Taking the scaling relevant for a $\mathrm{He}$ WD (van Haaften et al. 2012, see their eqn. [25], with $M_{p}=5.66 \times 10^{-4} M_{\odot}$ ), we tabulate values for $x=\log M_{\mathrm{WD}}$ and $y=\log R_{\mathrm{WD}}$ over the range of interest, $0.5<m_{1}<0.7$, and fit a least squares power law $R_{\mathrm{WD}}=10^{8.80} \mathrm{~cm} m_{1}-0.62$. Over the fit range this relation gives a maximum deviation $<1.5 \%$ from the Eggleton scaling.

Applying these conversions to the scalings given in $\mathrm{KLDH}$ (and ignoring the weak $\alpha$ dependencies) we obtain scalings for $\dot{M}_{T, 1}$ and $\dot{M}_{T, 2}$ relevant for their three compositions (i) $Y=1$, (ii) $Y=0.98, Z=0.02$, and (iii) $Y=0.96, Z=$

\footnotetext{
${ }^{5}$ We use $M_{1}$ and $M_{2}$ to refer to the primary and secondary masses in cgs units, and $m_{1}$ and $m_{2}$ to indicate $M / M_{\odot}$.
}

TABLE 1

KLDH-BASED COEFFICIENTS FOR LOCAL EXTREMA

\begin{tabular}{ccccccc}
\hline \hline comp. & $\begin{array}{c}\dot{M}_{T, 1,0} \\
\left(M_{\odot} \mathrm{yr}^{-1}\right)\end{array}$ & $\begin{array}{c}\dot{M}_{T, 2,0} \\
\left(M_{\odot} \mathrm{yr}^{-1}\right)\end{array}$ & $\epsilon_{1 m 1}$ & $\epsilon_{2 m 1}$ & $\epsilon_{q}$ & $\epsilon_{p}$ \\
\hline (i) & $10^{-10.89}$ & $10^{-8.60}$ & -2.54 & 0.16 & 0.89 & 2.0 \\
(ii) & $10^{-11.04}$ & $10^{-8.82}$ & -2.50 & 0.16 & 0.89 & 1.99 \\
(iii) & $10^{-11.10}$ & $10^{-8.93}$ & -2.49 & 0.16 & 0.88 & 1.97 \\
\hline
\end{tabular}

0.04. Adopting the general forms

$$
\begin{aligned}
& \dot{M}_{T, 1}=\dot{M}_{T, 1,0} m_{1}\left(P_{2}\right)^{\epsilon_{1 m 1}} \\
& \dot{M}_{T, 2}=\dot{M}_{T, 2,0} m_{1}\left(P_{1}\right)^{\epsilon_{2 m 1}}(1+q)^{\epsilon_{q}}\left(\frac{P_{\text {orb }}}{1000 \mathrm{~s}}\right)^{\epsilon_{p}},
\end{aligned}
$$

we may now calculate the coefficients. These are given in Table 1.

Levitan et al. (2015) presents a list of AM CVn systems ordered by $P_{\text {orb }}$, with outburst properties indicated. The rate of mass transfer $\dot{M}_{T}$ decreases sharply with $P_{\text {orb }}$, so that the shortest $P_{\text {orb }}$ systems have disks in permanent outburst, those with intermediate $P_{\text {orb }}$ exhibit outbursts, and those with the longest $P_{\text {orb }}$ have disks in permanent low states. The dividing point between high state systems and outbursting systems lies at $P_{1} \approx 20 \mathrm{~min}$. The dividing point between outbursting systems and low state systems, $P_{2}$, is not as straightforward. A block of systems starting at $P_{\text {orb }}=44.3$ min are listed as not showing outburst 6 , but two systems among these do show outbursts, at $P_{\text {orb }}=47.3$ and $48.3 \mathrm{~min}$. The expressions for $\dot{M}_{T, 1}$ indicate a steep inverse scaling with $m_{1}$, therefore it seems probable that these systems have somewhat high primary masses. Therefore we set $P_{2}=44 \mathrm{~min}$. Furthermore we adopt $m_{1}\left(P_{1}\right)=m_{1}\left(P_{2}\right)=0.6$. We note that the $m_{1}$ values for AM CVn systems are not well-constrained observationally from dynamical measurements.

We may now use the observed instability strip for AM CVn systems to constrain the secondary mass transfer rate $\dot{M}_{T}$. Let us assume a power law $\dot{M}_{T}=A\left(P_{\text {orb }} / 1000 \mathrm{~s}\right)^{n}$. For simplicity we adopt the convention that $\dot{M}_{T}>0$. Since we expect $\dot{M}_{T}$ to decrease with orbital period, we set $\dot{M}_{T, 1}=$ $\dot{M}_{T}\left(P_{2}\right)$ and $\dot{M}_{T, 2}=\dot{M}_{T}\left(P_{1}\right)$, which gives two equations in two unknowns. We may solve for the normalization constant $A$ and dependence on orbital period $n=d \ln \dot{M}_{T} / d \ln P_{\text {orb }}$. This yields the general solution

$$
\begin{aligned}
& n=\frac{\log \left[\left(\dot{M}_{T, 2,0} / \dot{M}_{T, 1,0}\right) g\left(P_{1} / 1000 \mathrm{~s}\right)^{\epsilon_{p}}\right]}{\log \left(P_{1} / P_{2}\right)} \\
& A=\dot{M}_{T, 1,0} m_{1}\left(P_{2}\right)^{\epsilon_{1 m 1}}\left(\frac{P_{2}}{1000 \mathrm{~s}}\right)^{-n},
\end{aligned}
$$

where

$$
g=(1+q)^{\epsilon_{q}} m_{1}^{\epsilon_{2 m 1}-\epsilon_{1 m 1}} .
$$

Table 2 gives the values for $A$ and $n$ for the three KLDH compositions, adopting $P_{1}=20 \mathrm{~min}, P_{2}=44 \mathrm{~min}$, and $m_{1}=0.6 \pm 0.05$. The magnitude of the putative assigned error on $m_{1}$ was propagated through to $n$ and $A$ in order to indicate the strength of the dependency. The main uncertainty entering into $n$ and $A$ is the assumed primary mass. For in-

\footnotetext{
6 There is also a system within the instability zone at 35.2 min indicated as not having outbursts. We disregard it on the assumption it must be anomalous in some way, or its outbursts may have been missed.
} 
TABLE 2

COEFFicients FOR $\dot{M}_{T}=A\left(P_{\text {orb }} / 1000 \mathrm{~s}\right)^{n}$ WITH $m_{1}=0.6 \pm 0.05$

\begin{tabular}{ccc}
\hline \hline composition & $A\left(M_{\odot} \mathrm{yr}^{-1}\right)$ & $n$ \\
\hline (i) & $8.82 \pm 0.6 \times 10^{-9}$ & $-5.38 \pm 0.3$ \\
(ii) & $5.27 \pm 0.4 \times 10^{-9}$ & $-5.23 \pm 0.3$ \\
(iii) & $3.88 \pm 0.3 \times 10^{-9}$ & $-5.06 \pm 0.3$ \\
\hline
\end{tabular}

stance, taking $m_{1}=1$ would give $n \simeq-7$, a much steeper $\dot{M}_{T}\left(P_{\text {orb }}\right)$ relation.

Since precise abundances for AM CVns as a group are not known, we adopt a representative fiducial scaling $\dot{M}_{T} \simeq$ $5 \times 10^{-9} M_{\odot} \mathrm{yr}^{-1}\left(P_{\mathrm{orb}} / 1000 \mathrm{~s}\right)^{-5.2}$, relevant for the middle range of scalings given in $\mathrm{KLDH}$.

\subsection{Considerations from AM CVn Binary Evolution}

How does this $\dot{M}_{T}$ law compare to theoretical expectations? As mentioned above, in AM CVn systems the driving of the mass transfer is most likely the angular momentum loss due to GWR. For that case one can derive the expected scaling of mass transfer rate with orbital period as is done in Warner (1995b). We begin by expressing the mass transfer rate in terms of the angular momentum loss

$$
\frac{\dot{M}_{2}}{M_{2}} \propto\left(\frac{\dot{J}}{J_{\text {orb }}}\right)_{\mathrm{GWR}} \propto \frac{M_{1} M_{2}\left(M_{1}+M_{2}\right)}{a^{4}}
$$

(e.g., see Savonije et al. 1986; Marsh et al. 2004; eqn. [6] of van Haaften et al 2012), where $\dot{M}_{2}=-\dot{M}_{T}$. Assuming $M_{1}$ remains constant and $M_{2} \ll M_{1}$ and using Kepler's law to replace orbital separation with orbital period, we find

$$
\frac{\dot{M}_{2}}{M_{2}} \propto M_{2} P_{\text {orb }}^{-8 / 3}
$$

We then can use the fact that the size of the Roche lobe for given period depends only very weakly on the mass of the accretor (the well known period-mean density relation) to find how $P_{\text {orb }}$ scales with $M_{2}$, given the mass-radius relation for the donor $R_{2} \propto M_{2}^{\zeta}$. Hence

$$
P_{\text {orb }} \propto\left(\frac{R_{2}^{3}}{M_{2}}\right)^{1 / 2} \propto M_{2}^{(3 \zeta-1) / 2}
$$

and therefore

$$
\dot{M}_{2} \propto M_{2}^{2} P_{\text {orb }}^{-8 / 3} \propto P_{\text {orb }}^{4 /(3 \zeta-1)-8 / 3} .
$$

For $\zeta=-1 / 3$ we find an exponent] $n=-14 / 3$. In van Haaften et al. (2012) the values of $\zeta$ are plotted in their Figure 2. For low masses $\zeta>-1 / 3$, yielding a larger absolute value of the exponent. Indeed, the fit they make to the dependence of mass transfer rate on orbital period (their Appendix A) gives $n=-5.32$ for a $1.4 M_{\odot}$ accretor. Warner (1995b) uses $\zeta=-0.19$, based on Savonije et al. (1986), and finds $n=-5.21$. We conclude that the exponent for the scaling we infer from the DIM based on the observed instability strip for AM CVns is in good agreement with expectations from stellar structure if we adopt $m_{1} \simeq 0.6$.

\footnotetext{
7 Note that there is an exponent " -1 " missing in eqn. (4) of Warner (1995b); for $\zeta=-1 / 3$ the donor mass and orbital period scale inversely, not linearly.
}

\section{RECURRENCE TIME FOR OUTBURSTS}

For 11 of the 32 AM CVn systems with known orbital periods in their study, Levitan et al (2015) have enough coverage to make quantitative statements about their outburst properties. They find a relation for the recurrence time for outbursts

$$
t_{\text {recur }}=1.46 \mathrm{~d}\left(\frac{P_{\text {orb }}}{1000 \mathrm{~s}}\right)^{7.35}+24.7 \mathrm{~d} .
$$

They only include superoutbursts. Their relation (see their Figure 12a) is much tighter than the comparable plot for DNe above the minimum period (e.g., see Figures 2-4 of CSW; Figure 11 of Patterson 2011).

What is the expectation from DIM for the recurrence time and is it consistent with this scaling?

CSW formulated an analytical expression for $t_{\text {recur. Their }}$ full complexity is not needed, and indeed one can take a rather simple approach. Regardless of whether one subscribes to the thermal-tidal instability for superoutbursts or the plain DIM (Osaki \& Kato 2013), the normal outbursts in a system exhibiting both normal outbursts and superoutbursts represent a tiny fraction of the mass budget. Therefore a good approximation is that superoutbursts are the only outbursts, and that during a superoutburst essentially all the mass stored in the cold state is accreted. The recurrence time is then simply

$$
t_{\text {recur }}=\frac{\Delta M_{\text {cold }}}{\dot{M}_{T}}=\frac{f \Delta M_{\max }}{\dot{M}_{T}}=\frac{f \int 2 \pi r d r \Sigma_{\max }(r, \alpha)}{\dot{M}_{T}},
$$

where $\Delta M_{\max }$ is the maximum mass that could be stored in quiescence and $f$ is the fraction the disk is filled, relative to this maximum.

For specificity we adopt the middle of the three compositions considered by KLDH. Their $\Sigma_{\max }$ for $Y=0.98$, $Z=0.02$ is

$$
\Sigma_{\max }=612 \mathrm{~g} \mathrm{~cm}^{-2} \alpha_{c-1}{ }^{-0.82} r_{10}{ }^{1.10} m_{1}{ }^{-0.37},
$$

where $\alpha_{c-1}$ is the value of $\alpha$ in the low state of the disk, $\alpha_{\text {cold }}$, normalized to 0.1 . Hence

$$
\begin{array}{r}
\Delta M_{\max }=1.04 \times 10^{-9} M_{\odot} \alpha_{c-1}{ }^{-0.82} m_{1}^{0.85} \\
(1+q)^{1.03}\left(\frac{P_{\text {orb }}}{1000 \mathrm{~s}}\right)^{2.31} .
\end{array}
$$

Evaluating the recurrence time gives

$$
\begin{aligned}
t_{\text {recur }} & =7.59 \mathrm{~d} f_{-1} \alpha_{c-1}{ }^{-0.82} m_{1}^{0.85}(1+q)^{1.03}\left(\frac{P_{\text {orb }}}{1000 \mathrm{~s}}\right)^{7.51} \\
& =4.92 \mathrm{~d}\left(\frac{P_{\text {orb }}}{1000 \mathrm{~s}}\right)^{7.51}
\end{aligned}
$$

for $m_{1}=0.6$, where $f_{-1}=f / 0.1=\alpha_{c-1}=1$. Thus for a mass transfer rate with $n=d \ln \dot{M}_{T} / d \ln P_{\text {orb }}=-5.2$, the simplest recurrence time scaling gives $t_{\text {recur }} \propto P_{\text {orb }}{ }^{7.5}$, which is close to the observed relation.

\section{DURATION TIME FOR OUTBURSTS}

For the AM CVn superoutburst durations Levitan et al. (2015) find

$$
t_{\mathrm{dur}}=0.89 \mathrm{~d}\left(\frac{P}{1000 \mathrm{~s}}\right)^{4.54}+10.6 \mathrm{~d} .
$$


This relation appears to be less reliable than their $t_{\text {recur }}\left(P_{\text {orb }}\right)$ relation: for the four longest period systems only upper limits are given. In fact, for the other six systems, those with $22 \mathrm{~min}$ $<P_{\text {orb }}<29$ min, their data are consistent with $t_{\mathrm{dur}}\left(P_{\text {orb }}\right)$ being constant with orbital period. We note that the four outburst durations given as upper limits in Table 2 of Levitan et al. are plotted as actual values in their Figure 12, panel 3, and enter into their power law fitting. As noted in their Appendix A, for the four systems with only upper limits $(U L)_{i}$ on $t_{\mathrm{dur} i}$ they arbitrarily take $t_{\mathrm{dur} i}=0.75(U L)_{i}$, with error $0.25(U L)_{i}$.

What would one expect from theory?

There are several ways to construct an outburst duration timescale for superoutbursts. During a long outburst with a "viscous plateau", the cooling front cannot propagate due to excess surface density at the outer disk edge, relative to $\Sigma_{\min }$, therefore the only option is for accretion onto the primary so that $\Sigma\left(r_{\text {outer }}\right)$ is gradually reduced. Therefore it is reasonable to consider the viscous time scale in the outer disk in the hot state as an approximation to the superoutburst duration.

We follow the method given in King \& Pringle (2009). They estimate a local peak accretion rate during outburst as $\simeq 2 \Delta M_{\text {hot }} / t_{\text {visc }}$, where $\Delta M_{\text {hot }}$ is the mass of the hot disk. The outbursting disk mass is determined by that stored in quiescence, $\Delta M_{\text {hot }}=\Delta M_{\text {cold }}$. This eliminates $\dot{M}$ from the standard equations given in Frank, King, \& Raine (2002), which relate the locally defined viscous time to $\alpha, r$, and $\dot{M}$. Using the outer disk edge for $r$ and relating it to orbital period as previously gives

$$
t_{\text {visc }}=15.1 \mathrm{~d} \alpha_{h-1}^{-0.8} m_{1}^{0.34}(1+q)^{0.16}\left(\frac{P_{\text {orb }}}{1000 \mathrm{~s}}\right)^{0.36}
$$

where $\alpha_{h-1}=\alpha_{\text {hot }} / 0.1$.

This is much flatter than the Levitan et al. relation for $t_{\mathrm{dur}}\left(P_{\mathrm{orb}}\right)$, but their fitted relation is dominated by including upper limits for $t_{\text {dur }}$ (for systems with $P_{\text {orb }}>30 \mathrm{~min}$ ) as part of their fit, which makes a direct comparison with theory problematic.

\section{DISCUSSION AND CONCLUSION}

Previous workers have examined the stability properties of AM CVn systems in the context of expectations from the DIM, e.g., TO97 (see their Fig. 4), Nelemans (2005, see his Figs. 1 and 6), LDK (see their Fig. 3), and KLDH (see their Fig. 3). The last work, KLDH, has provided the most complete study to date. Although they do not provide explicit formulae between the critical mass transfer rates and orbital period as in TO97, KLDH plot them in their Fig. 3, as do the aforementioned studies. Our main difference with KLDH is that for $\dot{M}_{T, 1}$ we also take into account the zero torque boundary condition at the inner edge of the disk (following TO97), in addition to just considering the primary mass. However, $\dot{M}_{T, 1}$ depends steeply on the primary mass $\left(\underset{\sim}{\sim} m_{1}^{-2.5}\right.$ ), and this uncertainty will likely dominate that associated with the boundary condition refinement. KLDH plot in their Fig. 3 the expected secular $\dot{M}_{T}$ versus $P_{\text {orb }}$ from stellar evolutionary models, and find consistency with the DIM in terms of stability of observed systems. Thus the difference between our study and KLDH is one of perspective: KLDH combine observations with evolutionary models to show that AM CVn outbursts can be explained by the DIM, whereas we assume that the DIM is correct in order to derive $\dot{M}_{T}\left(P_{\text {orb }}\right)$, and then explore the ramifications of the derived law vis a vis not only $\dot{M}_{T}\left(P_{\text {orb }}\right)$ predicted from evolutionary models, but also the resultant outburst properties versus $P_{\text {orb }}$.

In summary, we apply the DIM model to the recent results on AM CVn systems obtained by Levitan et al. (2015), using scalings for the helium-rich accretion disks in such systems taken from Kotko et al. (2012). The orbital periods defining the edges of the instability strip $P_{1}$ and $P_{2}$ permit us to infer a mean secondary mass transfer rate $\dot{M}_{T} \simeq$ $5 \times 10^{-9} M_{\odot} \mathrm{yr}^{-1}\left(P_{\mathrm{orb}} / 1000 \mathrm{~s}\right)^{-5.2}$. Our finding of a steep inverse dependence $n \simeq-5.2$ is consistent with theoretical expectations, but our result is dependent on taking $m_{1} \simeq 0.6$; higher $m_{1}$ steepens the relation. Treating $m_{1}$ as a variable and all other parameters on the right hand side of equation (7) as constant we may write $n \approx-5.23-7.75 \log \left(m_{1} / 0.6\right)$ for $Y=0.98, Z=0.02$. The largest uncertainty in $n$ enters via $m_{1}$.

We emphasize that the power-law form for $P_{\text {orb }}$ is not an outcome of our analysis but an assumption, valid in our application only over $20 \mathrm{~min} \lesssim P_{\text {orb }} \lesssim 45 \mathrm{~min}$. However, our inferred $P_{\text {orb }}$ values at 20 and $45 \mathrm{~min}$ are in line with those estimated for stable systems with similar orbital periods (KLDH, see their Fig. 3). We note that the precise values of $P_{\text {orb }}$ defining the edge of the instability strip, $P_{1}$ and $P_{2}$, also affect $n$ and $A$, although not as strongly as $m_{1}$. Lastly, $n$ and $A$ are only weakly dependent on the composition of the gas and the viscosity parameter $\alpha$; these two factors enter via $\dot{M}_{T, 2,0} / \dot{M}_{T, 1,0}$ (see eqn. [7]). This ratio is relatively insensitive to composition and virtually independent of $\alpha$. The full dependencies of $n$ and $A$ on all input parameters are given in eqs. (7)-(9).

The simplest possible theoretical expression for the recurrence time from the DIM gives $t_{\text {recur }} \propto r_{\text {outer }}^{3.1} / \dot{M}_{T} \propto$ $P_{\text {orb }}{ }^{2.31} \dot{M}_{T}^{-1}$, so that $\left(d \ln t_{\text {recur }} / d \ln P_{\text {orb }}\right)=2.31-$ $(-5.2) \simeq 7.5$, close to the value 7.35 found by Levitan et al. (2015). However, a larger assumed value of $m_{1}$ in our model would increase the exponent. Thus, if the DIM is relevant for AM CVn outbursts, the primaries must have masses $m_{1} \simeq 0.6$. The Levitan et al. constraint on the outburst duration $\propto P_{\text {orb }}^{4.54}$ appears to be dominated by incompleteness for the upper half of their range in orbital period. Our theoretical prediction is that a more complete time sampling of AM CVn outbursts, especially at longer orbital period, will ultimately reveal a much flatter law $t_{\mathrm{dur}} \propto P_{\text {orb }}^{0.4}$.

We thank Joe Patterson for organizing a small CV workshop at Columbia University in Fall of 2014 which provided a stimulating environment for discussion. We also thank the anonymous referee whose comments improved the paper. 


\section{REFERENCES}

Cannizzo, J. K. 1984, Nature, 311, 443

Cannizzo, J. K. 1993a, in Accretion Disks in Compact Stellar Systems, ed. J. C. Wheeler (Singapore: World Scientific), 6

Cannizzo, J. K. 1993b, ApJ, 419, 318

Cannizzo, J. K., Shafter, A. W., \& Wheeler, J. C. 1988, ApJ, 333, 227 (CSW)

Cannizzo, J. K., \& Wheeler, J. C. 1984, ApJS, 55, 367

Deloye, C. J., Bildsten, L., \& Nelemans, G. 2005, ApJ, 624, 934

El-Khoury, W., \& Wickramasinghe, D. 2000, A\&A, 358, 154

Faulkner, J., Flannery, B. P., \& Warner, B. 1972, ApJ, 175, L79

Frank, J., King, A. R., \& Raine, D. J. 2002, Accretion Power in Astrophysics, 3rd ed. (Cambridge: Cambridge Univ. Press)

Groot, P. J., Nelemans, G., Steeghs, D., \& Marsh, T. R. 2001, ApJ, 558, L123

King, A. R., \& Pringle, J. E. 2009, MNRAS, 397, L51

Knigge, C., Baraffe, I., \& Patterson, J. 2011, ApJS, 194, 28 (KBP)

Kotko, I., Lasota, J.-P., Dubus, G., \& Hameury, J.-M. 2012, A\&A, 544, A13 (KLDH)

Lasota, J.-P. 2001, New Astron. Rev., 45, 449

Lasota, J.-P., Dubus, G., \& Kruk, K. 2008, A\&A, 486, 523 (LDK)

Levitan, D., Groot, P. J., Prince, T. A., Kulkarni, S. R., Laher, R., Ofek, E. O. Sesar, B., \& Surace, J. 2015, MNRAS, 446, 391

Marsh, T. R., Nelemans, G., \& Steeghs, D. 2004, MNRAS, 350, 113

Menou, K., Perna, R., \& Hernquist, L. 2002, ApJ, 564, L81

Meyer, F. \& Meyer-Hofmeister, E. 1981, A\&A, 104, L10

Nather, R. E., Robinson, E. L., \& Stover, R. J. 1981, ApJ, 244, 269
Nelemans, G. 2005, in The Astrophysics of Cataclysmic Variables and Related Objects, eds. J.-M. Hameury, \& J.-P. Lasota, Proc. ASP Conf., 330, 27

Osaki, Y., \& Kato, T. 2013, PASJ, 65, 50

Patterson, J. P. 2011, MNRAS, 411, 2695

Patterson, J., Halpern, J., \& Shambrook, A. 1993, ApJ, 419, 803

Ramsay, G., Barclay, T., Steeghs, D., Wheatley, P. J., Hakala, P., Kotko, I., \& Rosen, S. 2012, MNRAS, 419, 2836

Rappaport, S., Ma, C. P., Joss, P. C., \& Nelson, L. A. 1987, ApJ, 322, 842

Savonije, G. J., de Kool, M., \& van den Heuvel, E. P. J. 1986, A\&A, 155, 51

Shakura, N. I., \& Sunyaev, R. A. 1973, A\&A, 24, 337

Smak, J. 1983a, Acta Astron., 33, 333

Smak, J. 1983b, ApJ, 272, 234

Smak, J. 1984, Acta Astr., 34, 161

Solheim, J.-E. 2010, PASP, 122, 1133

Tsugawa, M., \& Osaki, Y. 1997, PASJ, 49, 75 (TO97)

van Haaften, L. M., Nelemans, G., Voss, R., Wood, M. A., \& Kuijpers, J. 2012, A\&A, 537, A104

van Paradijs, J. 1983, A\&A, 125, L16

Warner, B. 1995a, Cataclysmic Variable Stars (Cambridge: Cambridge Univ. Press)

Warner, B. 1995b, Ap\&SS, 225, 249

Warner, B., \& Robinson, E. L. 1972, MNRAS, 159, 101 